\title{
BMJ Open Health-related quality of life in patients with diabetic foot ulceration: study protocol for adaptation and validation of patient-reported outcome measurements (PROMs) in Dutch- speaking patients
}

\author{
Wahid Rezaie (D) , ${ }^{1}$ Flora Lusendi, ${ }^{2}$ Kris Doggen, ${ }^{2}$ Giovanni Matricali, ${ }^{3}$ \\ Frank Nobels ${ }^{4}$
}

To cite: Rezaie W, Lusendi F, Doggen $\mathrm{K}$, et al. Health-related quality of life in patients with diabetic foot ulceration: study protocol for adaptation and validation of patient-reported outcome measurements (PROMs) in Dutch-speaking patients. BMJ Open 2019;9:e034491. doi:10.1136/ bmjopen-2019-034491

- Prepublication history for this paper is available online. To view these files, please visit the journal online (http://dx.doi. org/10.1136/bmjopen-2019034491).

Received 25 September 2019 Revised 24 November 2019 Accepted 04 December 2019

Check for updates

(c) Author(s) (or their employer(s)) 2019. Re-use permitted under CC BY-NC. No commercial re-use. See rights and permissions. Published by BMJ.

For numbered affiliations see end of article.

Correspondence to

Dr Wahid Rezaie;

wrezaie@me.com

\section{ABSTRACT}

Introduction Diabetic foot ulceration (DFU) is a common late-stage complication of diabetes with a large impact on health status and quality of life. Patient-reported outcome measures (PROMs) provide a standardised method of obtaining patients' views on their well-being. The DFU Scale Short Form (DFS-SF) is a validated disease-specific PROM for measuring health-related quality of life among DFU patients. The Lower Extremity Functional Scale (LEFS) is another PROM that can be used to measure physical functioning in patients with lower extremity disorders. The LEFS is not yet validated for DFU. Both instruments are not validated in the Dutch language. The purpose of this study is to culturally adapt and validate the DFS-SF and LEFS questionnaires for Belgian Dutch-speaking patients with DFU.

Methods and analysis This study will be conducted as a monocentre observational cohort study in DFU patients presenting at a hospital-based multidisciplinary diabetic foot clinic. Data will be collected from the medical electronic files and from DFS-SF, LEFS and five-level EuroQol five-dimension questionnaires that will be presented to the patients at defined time points. Reproducibility, internal consistency, floor and ceiling effects, construct validity and responsiveness will be assessed for the DFS-SF and LEFS.

Ethics and dissemination The study protocol has been approved by the Medical Ethics Committee of Onze-LieveVrouw Hospital (Aalst, Belgium). The results of the study will be disseminated through peer-reviewed publications and conference presentations.

\section{INTRODUCTION}

Diabetic foot ulceration (DFU) is a common late-stage complication of diabetes, with $19 \%-34 \%$ of individuals with diabetes experiencing a DFU in their lifetime. ${ }^{1}$ These ulcers have a large impact on the health status and quality of life of the affected patients ${ }^{2}$ and are associated with major healthcare
Strengths and limitations of this study

- This is the first study that aims to validate the Diabetic Foot Ulcer Scale Short Form and the Lower Extremity Functional Scale into Dutch.

- The proposed study conforms to the COnsensusbased Standards for the selection of health Measurement Instruments guidelines.

- This is a prospective monocentre observational study, which will be conducted in a high-volume hospital-based multidisciplinary diabetic foot clinic.

- Although the study will be applicable in Dutchspeaking regions in Belgium and the Netherlands, it is also relevant and informative for other regions.

- The study is limited to adults who have a severe DFU (Wagner $\geq 2$ ) and who were able to ambulate prior to developing DFU.

consumption and high costs. ${ }^{34}$ They require intensive multidisciplinary treatment, and hospital admission rates for DFU exceed the rates for congestive heart failure, renal disease, depression and most forms of cancer. ${ }^{5}$

In recent decades, understanding of the pathogenesis, treatment and prevention has improved. ${ }^{1}$ In contrast, our knowledge of the impact of a foot ulcer on the quality of life of a diabetic patient is limited. This is nevertheless important, because the success of a long-term treatment and the compliance with preventive measures depend to a large extent on the mental resilience and motivation of the patient and on the patient's participation in his treatment.

Patient-reported outcome measures (PROMs) are essential in evaluating quality of life and functional outcomes after application of a treatment as well as in clinical 
research. ${ }^{6}$ PROMs can also be used in quality assessment of care across hospitals. Belgian hospital-based diabetes foot clinics are obligated to participate in IQED-Foot (Initiative for Quality improvement and Epidemiology in multidisciplinary Diabetic Foot Clinics) which includes audit-feedback cycles covering both processes and outcomes of care. ${ }^{78}$ Data on quality of life are not yet included in this initiative but may be used as a quality-ofcare indicator for IQED-Foot in the future.

Literature on PROMs for DFU is limited. In a recent systematic review, based on an extensive search of available literature until September 2018, only 11 studies of appropriate quality were found. ${ }^{9}$ A broader systematic review covering PROMs in the area of foot and ankle disease, also including studies on DFU, confirmed that the measurement properties of many instruments have been insufficiently studied and reported. ${ }^{10} \mathrm{~A}$ valid Dutch version of a disease-specific PROM for DFU is currently not available.

A systematic review of the literature showed that the Diabetic Foot Ulcer Scale (DFS) is the most frequently used disease-specific PROM for measuring health-related quality of life (HRQoL) among diabetic foot patients. ${ }^{11}$ This instrument was developed by using semi-structured interviews and focus groups of patients with DFUs and their caregivers. ${ }^{12}$ It has shown internal consistency, reliability, validity and responsiveness to wound severity and healing. A shortened version, the DFS Short Form (DFS-SF), showed similar robustness and responsiveness compared with its original version, ${ }^{13}$ and is with 29 questions a more 'user-friendly' tool for everyday clinical practice. Despite its translation to several languages and subsequent use in studies ${ }^{14-19}$ its measurement properties have, however, not yet been studied and reported across all the relevant dimensions of the COnsensus-based Standards for the selection of health Measurement Instruments (COSMIN) Taxonomy of Measurement Properties. ${ }^{20}$ There are Dutch translations of DFS for Belgium and of DFS-SF for the Netherlands available which have undergone a full linguistic process according to the recognised methodology of translation as described in Linguistic Validation Manual for Health Outcome Assessments. ${ }^{21}$ This manual is the official guideline of the Mapi Institute, owner of the copyright on the questionnaire. However, the measurement properties of these questionnaires in a DFU population have not been evaluated yet in the Dutch language versions.

PROMs on physical functioning are available for lower extremity-related pathologies such as osteoarthritic conditions. However, most are not able to differentiate pain and function. ${ }^{22}$ The Lower Extremity Functional Scale (LEFS) can distinguish between pain and function in a wide variety of disorders. ${ }^{23}{ }^{24}$ The LEFS is validated in several languages, including in Dutch for patients with osteoarthritis. ${ }^{25}$ To our knowledge, the LEFS is not yet validated for assessing functional impairment of foot and ankle in diabetic foot conditions.

In addition to the disease-specific PROMs measuring HRQoL and physical functioning generic HRQoL instruments are also available. They collect information on the general quality of life. They can be compared with data issued from the general population and across pathologies. The EuroQol five dimension (EQ-5D) questionnaire is such a standardised instrument consisting of five domains and a summary index. The five-level EQ-5D version (EQ-5D-5L) was introduced by the EuroQol Group in 2009 to improve the instrument's sensitivity and to reduce ceiling effects. The EQ-5D-5L is among the most commonly used questionnaires for evaluating HRQoL. ${ }^{26}$ Several studies support its validity and reliability in many health-related conditions such as DFU in different languages. ${ }^{27-29}$

The aims of the study are:.

- To adapt and validate the Dutch DFS-SF questionnaire for evaluating the disease-specific HRQoL of diabetic patients with a DFU.

- To validate the Dutch LEFS questionnaire for evaluating the physical functioning of diabetic patients with a DFU.

\section{METHODS AND ANALYSIS}

\section{Study design}

This study will be conducted as a monocentre observational cohort study in Onze-Lieve-Vrouw (OLV) Hospital Aalst. Data will be collected from the medical electronic files and from DFS-SF, LEFS and EQ-5D-5L questionnaires that will be presented to the patients at defined time points. Additional questions will be asked about loss of productivity (work absenteeism), financial burden (out of pocket expenses) and satisfaction with the medical treatment. There are no medical interventions, nor extra visits or laboratory tests planned outside the normal clinical routine.

The study will consist of the following three parts.

\section{Part 1: preparing the questionnaires}

Minor linguistic adaptations will be made by research team to the Netherlands Dutch DFS-SF and LEFS questionnaires to adapt them for Belgian native Dutch-speaking patients.

To clear out linguistic ambiguities, 10 patients will be asked if they understand the questions and are able to provide answers. Based on their remarks small changes will be made if necessary. Care will be taken to make only small linguistic changes that do not change the content of the questionnaires. When changes are needed, another group of 10 patients will be asked if they understand the questions and are able to provide answers. If this is the case, no further adaption to the questionnaire will be necessary. If there are ambiguities for the patients, the adaptation procedure of the questionnaire will be repeated until no changes are needed. At this point, the questionnaires are final and will not be changed anymore during the entire study.

\section{Part 2: testing reproducibility and measurements properties}

In order to determine the reproducibility (ie, test-retest reliability) of the DFS-SF and LEFS questionnaires, 50 study participants will be asked to complete the questionnaires twice. This time interval needs to be sufficiently 
short for the patient's foot condition to remain stable and sufficiently long to prevent remembrance of the answers to the first questionnaire. We consider an interval of 2-3 weeks between completing two questionnaires. Patients who underwent foot surgery or revascularisation of the leg in this time interval will be excluded.

Patients will be asked about their subjective feeling of presence or absence of change in their foot condition before completing the second questionnaire. They will be asked: 'Did the condition of your feet remain stable, compared with the last time you completed this questionnaire, yes or no?' Patients reporting a change will be excluded from the analysis. Patients reporting no change will be considered stable between the two measurements and are included for testing the reproducibility of the questionnaires.

\section{Part 3: testing measurement validity}

Recruitment will continue until a sample size of at least 100 patients is reached (see the Sample size considerations section) for testing the measurement validity of the questionnaires. It is estimated that this will take 2 years.

All questionnaires will be administered at the first presentation of patient on the diabetic foot clinic. The patients will be asked to complete the questionnaire again at moments of important treatment changes (eg, before and after surgical treatment) or 6 months after starting care (if the patient is still in follow-up) or at the moment of healing of a foot ulcer when it takes less than 6 months.

\section{Study population}

All consecutive patients attending the multidisciplinary outpatient clinic or being admitted at OLV Hospital diabetic foot clinic, and who meet the inclusion criteria, will be invited to participate. The recruitment of patients has started in April 2019. The inclusion of the patients expected to be completed by the end of 2020. The follow-up of patients will be ended in June 2021.

The inclusion criteria are:

- Adult patients $\geq 18$ years.

- Adequate comprehension of the Dutch to understand the questionnaires.

- Having a severe DFU (Wagner $\geq 2$ ).

- Attending the multidisciplinary outpatient diabetic foot clinic or admission to the OLV inpatient diabetic foot department.

- Able to provide written informed consent.

Patients with any of following criteria will be excluded from participation in the study:

- Patient was not able to ambulate prior to DFU (ie, bedridden or wheelchair-dependent).

- Cognitive dysfunction (which hampers the understanding of questionnaires).

\section{Scoring instruments}

The DFS-SF consists of six conceptual domains (leisure, physical health, dependence/daily life, negative emotions, worried about ulcers/feet and bothered by ulcer care).
The questionnaire comprises of 29 items with a 5-point Likert rating scale. To create subscale scores, all items are reverse-coded so that higher values indicate better QoL. ${ }^{13}$

The LEFS consists of 20 items. $^{24}{ }^{25}$ Items are rated on a 4-point scale, from 0 (extreme difficulty/unable to perform activity) to 4 (no difficulty). The maximum possible score is 80 points, indicating very high function. The minimum possible score is 0 points, indicating very low function.

The EQ-5D-5L provides a scoring system producing a 'health state profile' comprising of five different domains (mobility, self-care, usual activities, pain/discomfort and anxiety/depression). Each dimension is rated at five levels of severity: (no (level 1), slight (level 2), moderate (level 3), severe (level 4) and extreme problems/unable (level 5)). ${ }^{26}$

\section{Study setting}

The questionnaires will be delivered digitally on a tablet. This is the preferential mode of delivery.

The patients will be instructed on how to use the tablet. A research nurse will assist patients who find it difficult to use a tablet. The tablet will be provided to the patient before the consultation in the waiting room. A physician from the diabetic foot clinic will perform the physical examination.

The following data will be collected from the standard medical files, based on the variables included in IQEDFoot $^{78}$ : age, gender, comorbidities, diabetic foot-related variables, treatment-related variables and outcomes of treatment. The ulcer will be assessed using the PEDIS (Perfusion, Extent, Depth, Infection and Sensation) classification system. It includes five ulcer criteria, and each criterion is graded by the severity. ${ }^{30}$

\section{Study duration}

Patients will be followed for a total period of 6 months or until healing of an ulcer when this takes less time. The study will continue until the sample size has been obtained.

\section{Statistical analysis}

All data will be entered in a clinical database. The SAS V.9.4 will be used to perform statistical analyses. The missing values will not be imputed, as the raw data for individual items would be analysed. Normality of continuous data will be tested with the Shapiro-Wilk test. Continuous data will be reported as mean $\pm \mathrm{SE}$ (parametric) or median with percentiles (non-parametric) and categorical data as numbers with percentages. Categorical variables will be assessed using $\chi^{2}$ tests. Continuous data will be compared using Student's t-test (parametric data) or Mann-Whitney $\mathrm{U}$ test (non-parametric data).

\section{Evaluation of measurements properties of DFS-SF and LEFS}

The measurement properties of the Belgian-Dutch versions of the DFS-SF and LEFS will be assessed. The characteristics that will be assessed are based on the COSMIN Taxonomy of Measurement Properties ${ }^{20}$ and 
the criteria for adequacy that will be used are based on publications by Terwee $e t a l,{ }^{14}$ Mokkink $e t a l^{31}$ and Heinl et al. $^{32}$ If the COSMIN Study Design checklist ${ }^{33}$ is available before data collection ends, we will take into account any additional criteria mentioned in this checklist before starting the assessment of the measurement properties.

\section{Reproducibility}

Reproducibility consists of two parts: reliability and agreement. Reliability concerns the extent to which scores for patients who have not changed are the same for repeated measurement under several conditions. Evaluation of the test-retest reliability will be performed by calculating the intraclass correlation coefficient (ICC agreement) with a corresponding $95 \%$ CI. Reliability will be given a positive rating when the ICC is at least 0.70 in a sample size of 50 patients.

Agreement concerns the absolute measurement error, that is, how close the scores on repeated measures are. The degree of absolute agreement will be expressed as the SE of measurement (SEM agreement). This SEM equals the square root of the error variance of an analysis of variance analysis, including the systematic differences $\left(\mathrm{SEM}=(\text { variance patient }+ \text { variance residual })^{0.5}\right.$. A sample size of at least 50 patients is considered as adequate for the assessment of the agreement parameter, based on a general guideline by Altman. ${ }^{34}$

\section{Internal consistency}

Internal consistency is defined as the extent to which items in a (sub)scale are intercorrelated, thus measuring the same construct. The correlation between items on a (sub)scale will be evaluated by calculating Cronbach's $\alpha$ for every (sub)scale. Internal consistency is considered sufficient if the value for Cronbach's $\alpha$ is between 0.70 and 0.95 , provided that the scale is unidimensional. Regarding the number of subjects to include, rules-ofthumb vary from 4 to 10 subjects per variable, with a minimum number of 100 subjects to ensure stability of the variance-covariance matrix. ${ }^{34}$

\section{Floor and ceiling effects}

Floor and ceiling effects will be determined by calculating the number of individuals who obtained the lowest (floor) or highest (ceiling) possible scores and will be considered present if more than $15 \%$ of the respondents achieved the lowest or highest score in a sample size of at least 50 patients.

\section{Construct validity}

In order to evaluate the construct validity of DFU-FS and LEFS, we will use a set of hypotheses between the instruments scores (convergent validity) and the population characteristics or treatments (discriminative validity). In the literature, a positive rating for construct validity is given if hypotheses are specified in advance and at least $75 \%$ of the results are in correspondence with these hypotheses, in (sub)groups of at least 50 patients. ${ }^{35}$ Pearson's product-moment correlation coefficients (parametric data) or Spearman's r (rank correlation) coefficients (non-parametric data) will be calculated. Correlation coefficients above 0.6, between 0.6 and 0.3 and $<0.3$ will be considered high, moderate and low correlations, respectively. We will use Student's t-test (parametric data) or Mann-Whitney U test (nonparametric data) to compare two groups of patients based on their characteristics.

We propose the following hypotheses:

For the LEFS:

1. Patients with bilateral foot lesions (ulcer and/or active Charcot and/or previous leg amputation) demonstrate lower LEFS scores than patients with unilateral foot lesions.

2. Patients with only plantar foot ulcers demonstrate lower LEFS scores than patients with only non-plantar foot ulcers.

3. Patient with only toe wounds demonstrate higher LEFS scores than patients with wounds at other locations of the foot

4. There is a moderate negative correlation $(\mathrm{r}=0.3-0.6)$ between the LEFS score and the PEDIS score of the most severe foot ulcer.

5. Patients with bone involvement (PEDIS D3) demonstrate lower LEFS scores than patients without bone involvement.

6. Patients with peripheral artery disease (PEDIS P2 and 3) demonstrate lower LEFS scores than patients without peripheral artery disease (PEDIS P1).

For the DFS-SF:

1. Patients with bilateral foot lesions (ulcer, active Charcot and/or previous leg amputation) demonstrate lower DFS-SF scores than patients with unilateral foot lesions.

2. Patients with only plantar foot ulcers demonstrate lower DFS-SF scores than patients with only non-plantar foot ulcers.

3. Patients with only toe wounds demonstrate higher DFS-SF scores than patients with wounds at other locations of the foot.

4. There is a moderate negative correlation $(\mathrm{r}=0.3-0.6)$ between the DFS-SF score and the PEDIS score of the most severe foot ulcer.

5. Patients with bone involvement (PEDIS D3) demonstrate lower DFS-SF scores than patients without bone involvement.

6. Patients with peripheral artery disease (PEDIS P2 and 3) demonstrate lower DFS-SF scores than patients without peripheral artery disease (PEDIS P1).

\section{Criterion validity}

Criterion validity refers to the extent to which scores on a particular instrument relate to a gold standard. EQ5D has been used in several pivotal studies in patients with DFU. ${ }^{36-38}$ HRQoL scores will be measured at baseline and at 6 months (defined as 4-8 months). To evaluate the performance of DFS-SF, results of DFS-SF and EQ-5D will be correlated at the level of individual patients using Pearson or Spearman rank correlation techniques. In 
addition, changes in the different domains of DFS-SF will be compared with changes in EQ-5D.

\section{Responsiveness}

Responsiveness is defined as the ability of a questionnaire to detect clinically important changes over time, even if these changes are small. The DFS-SF and LEFS questionnaires will be administered at baseline, at 6 months and at the moment of healing. Changes over time will be analysed by calculating the differences between the three different time points for all questionnaire domains.

\section{Sample size considerations}

This study will test multiple hypotheses related to the following instruments: DFS-SF, LEFS and EQ-5D. Since the LEFS has not yet been used in the context of the diabetic foot, we cannot estimate sample size based on previously reported data using this instrument.

We estimated sample size at $\alpha=0.05$ and power of at least $80 \%$, as calculated by PROC POWER (SAS V.9.4).

In the context of construct validity, we identified the following previously reported cross-sectional associations.

First, Kontodimopoulos et $a \hat{l}^{8}$ reported associations of DFS-SF subscale scores with ulcer depth, graded as superficial, deep and up to bone. According to the group means and SD reported by the authors, and assuming the ulcer depth distribution observed in IQED-Foot (14\%, 56\% and $30 \%$ superficial, deep and up to bone, respectively ${ }^{7}$ ), the estimated sample size is 100 patients for all subscales to detect a significant overall difference between the three groups with one-way ANOVA.

Second, Macioch et $a l^{16}$ reported Spearman rank correlation coefficients between DFS-SF subscales and PEDIS ulcer classification. The strongest correlation was between the 'dependence/daily life' subscale and PEDIS perfusion grade, with a correlation coefficient of -0.312 . The estimated sample size to obtain a similar correlation coefficient was 78 patients.

Next, in the context of criterion validity (association of DFS-SF with EQ-5D), we identified no publications to which we could compare to estimate sample size.

Finally, in the context of responsiveness we identified a number of publications reporting mean and SD both for DFS-SF and EQ-5D, but the follow-up was not always 6 months. We estimated sample size for a paired t-test according to five different scenarios, depending on the extent to which within-patient scores were correlated over time: a correlation of $0,0.25,0.50,0.75$ or 1 .

Spanos $e t a l^{39}$ reported the change in DFS-SF subscale scores after 12 months. The estimated sample size range was 3-56 for the subscale with the lowest mean difference. The highest sample size is that needed in the hypothetical case that a patients' score was completely uncorrelated between 0 and 12 months.

Holman $e t a t^{0}$ reported the change in EQ-5D index score after 24 weeks. The estimated sample size range was 3-177.
Li $e t a l^{41}$ reported the change in EQ-5D index score after 12 weeks. We used data from the control group. The estimated sample size range was 7-123.

Siersma et $a t^{42}$ reported the change in EQ-5D index score after 12 months, separately for patients whose DFU healed within 6 months of follow-up, after 6 months of follow-up or whose DFU did not heal within 12 months. The estimated sample size range was 4-134, 3-129 and 3-720 for groups 1, 2 and 3, respectively.

In conclusion, a sample size of 100 patients seems sufficient to detect the most pronounced cross-sectional associations reported in the literature, among the set of associations that we are able to replicate in our study. With regard to the longitudinal change of HRQoL, there are no studies on the DFS-SF using a 6-month follow-up. Based on the use of EQ-5D, we can only compare with the study by Holman et al: in the worst case scenario, the sample size needed to detect a similar difference in HRQoL would be 177 patients. At a moderate withinpatient correlation of 0.5 , the sample size needed would be only 90 patients, well within the recruitment potential of the proposed study.

\section{Patient and public involvement}

It was not appropriate or possible to involve patients or the public in the design, or conduct, or reporting, or dissemination of our research.

\section{ETHICS AND DISSEMINATION}

Enrolment in this study does not have any influences on treatment. Verbal and written information including an information letter and an informed consent form will be given by a research physician or research nurses. Patients can always withdraw consent for inclusion of their data in the analysis.

The Investigator and the Participating Site will treat all information and data relating to the Study disclosed to Participating Site and/or Investigator in this Study as confidential and will not disclose such information to any third parties or use such information for any purpose other than the performance of the Study. The collection, processing and disclosure of personal data such as patient health and medical information are subject to compliance with General Data Protection Regulation (Directive 95/46/EC, Regulation (EU) 2016/679 of the European Parliament and of the Council of 27 April 2016 on the protection of natural persons with regard to the processing of personal data and on the free movement). The collected data will be coded. The clinical team will protect the data from disclosure outside the research.

The subject's name or other identifiers will be stored separately (site file) from their research data and replaced with a unique code to create a new identity for the subject. The research team will only have access to the coded research data.

The results of the study will be disseminated through peer-reviewed publications and conference presentations. 
If available at the time of publication, the results will be reported in accordance with the COSMIN Reporting checklist, which is a checklist that is still under development and will provide reporting guidelines for studies on measurement properties of PROMs. ${ }^{43}$

\section{Author affiliations}

${ }^{1}$ Orthopaedics \& Trauma, Multidisciplinary Diabetic Foot Clinic, Onze-Lieve-

Vrouwziekenhuis, Aalst, Belgium

${ }^{2}$ Health Services Research Unit, Belgian Scientific Institute of Public Health, Brussel, Belgium

${ }^{3}$ Multidisciplinary Diabetic Foot Clinic, KU Leuven Hospital, Leuven, Belgium ${ }^{4}$ Multidisciplinary Diabetic Foot Clinic, Onze-Lieve-Vrouwziekenhuis, Aalst, Belgium

Contributors WR and FN developed the study. WR was a principal investigator. WR, $\mathrm{FL}, \mathrm{KD}, \mathrm{GM}$ and FN were involved in writing the study protocol. WR, FL, KD, GM and $\mathrm{FN}$ will be involved in assessment of outcomes. $\mathrm{FL}$ and $\mathrm{KD}$ will perform statistical analysis of the study data. WR and FN drafted the manuscript.

Funding The authors have not declared a specific grant for this research from any funding agency in the public, commercial or not-for-profit sectors.

Competing interests None declared.

Patient consent for publication Not required.

Ethics approval The trial will be conducted in compliance with the principles of the Declaration of Helsinki (2013), the principles of GCP and in accordance with all applicable regulatory requirements. This protocol and related documents were approved by the Medical Ethics Committee of OLV Aalst (Belgian registration number B126201836509).

Provenance and peer review Not commissioned; externally peer reviewed.

Open access This is an open access article distributed in accordance with the Creative Commons Attribution Non Commercial (CC BY-NC 4.0) license, which permits others to distribute, remix, adapt, build upon this work non-commercially, and license their derivative works on different terms, provided the original work is properly cited, appropriate credit is given, any changes made indicated, and the use is non-commercial. See: http://creativecommons.org/licenses/by-nc/4.0/.

ORCID iD

Wahid Rezaie http://orcid.org/0000-0003-3535-280X

\section{REFERENCES}

1 Armstrong DG, Boulton AJM, Bus SA. Diabetic foot ulcers and their recurrence. N Engl J Med Overseas Ed 2017;376:2367-75.

2 Nabuurs-Franssen MH, Huijberts MSP, Nieuwenhuijzen Kruseman AC, et al. Health-related quality of life of diabetic foot ulcer patients and their caregivers. Diabetologia 2005;48:1906-10.

3 Prompers L, Huijberts M, Schaper N, et al. Resource utilisation and costs associated with the treatment of diabetic foot ulcers. prospective data from the Eurodiale study. Diabetologia 2008;51:1826-34.

4 Matricali GA, Dereymaeker G, Muls E, et al. Economic aspects of diabetic foot care in a multidisciplinary setting: a review. Diabetes Metab Res Rev 2007;23:339-47.

5 Skrepnek GH, Mills JL, Lavery LA, et al. Health care service and outcomes among an estimated 6.7 million ambulatory care diabetic foot cases in the U.S. Diabetes Care 2017;40:936-42.

6 Devlin NJ, Appleby J. Getting the most out of PROMs: putting health outcomes at the heart of NHS decision-making. London: BMJ Publishing Group, 2010.

7 Doggen K, Van Acker K, Beele H, et al. Implementation of a quality improvement initiative in Belgian diabetic foot clinics: feasibility and initial results. Diabetes Metab Res Rev 2014;30:435-43.

8 Morbach S, Kersken J, Lobmann R, et al. The German and Belgian accreditation models for diabetic foot services. Diabetes Metab Res Rev 2016;32 Suppl 1:318-25.

9 Ortega-Avila A, Cervera-Garvi P, Ramos-Petersen L, et al. Patientreported outcome measures for patients with diabetes mellitus associated with foot and ankle pathologies: a systematic review. $J$ Clin Med 2019;8:146-E146.

10 Jia Y, Huang H, Gagnier JJ. A systematic review of measurement properties of patient-reported outcome measures for use in patients with foot or ankle diseases. Qual Life Res 2017;26:1969-2010.
11 Hogg FRA, Peach G, Price P, et al. Measures of health-related quality of life in diabetes-related foot disease: a systematic review. Diabetologia 2012;55:552-65.

12 Abetz L, Sutton M, Brady L, et al. The diabetic foot ulcer scale (DFS): a quality of life instrument for use in clinical trials. Pract Diab Int 2002;19:167-75.

13 Bann CM, Fehnel SE, Gagnon DD. Development and validation of the Diabetic Foot Ulcer Scale-Short Form (DFS-SF). Pharmacoeconomics 2003;21:1277-90.

14 Terwee CB, Bot SDM, de Boer MR, et al. Quality criteria were proposed for measurement properties of health status questionnaires. J Clin Epidemiol 2007:60:34-42.

15 Kontodimopoulos N, Veniou A, Tentolouris N, et al. Validity and reliability of the Greek version of the Diabetic Foot Ulcer Scale-Short Form (DFS-SF). Hormones 2016;15:394-403.

16 Macioch T, Sobol E, Krakowiecki A, et al. Health related quality of life in patients with diabetic foot ulceration--translation and Polish adaptation of Diabetic Foot Ulcer Scale short form. Health Qual Life Outcomes 2017;15:15.

17 Sekhar MS, Thomas RR, Unnikrishnan MK, et al. Impact of diabetic foot ulcer on health-related quality of life: a cross-sectional study. Semin Vasc Surg 2015;28:165-71.

18 Lee Y-N. Translation and validation of the Korean version of the diabetic foot ulcer Scale-Short form. Int Wound J 2019;16 Suppl 1:3-12.

19 Alrub AA, Hyassat D, Khader YS, et al. Factors associated with health-related quality of life among Jordanian patients with diabetic foot ulcer. J Diabetes Res 2019;2019:4706720.

20 Mokkink LB, Terwee CB, Patrick DL, et al. The COSMIN study reached international consensus on taxonomy, terminology, and definitions of measurement properties for health-related patientreported outcomes. J Clin Epidemiol 2010;63:737-45.

21 Mapi Research Trust. Available: https://eprovide.mapi-trust.org

22 Terwee CB, van der Slikke RMA, van Lummel RC, et al. Self-reported physical functioning was more influenced by pain than performancebased physical functioning in knee-osteoarthritis patients. J Clin Epidemiol 2006;59:724-31.

23 Pua Y-H, Cowan SM, Wrigley TV, et al. The lower extremity functional scale could be an alternative to the Western Ontario and McMaster universities osteoarthritis index physical function scale. J Clin Epidemiol 2009;62:1103-11.

24 Binkley JM, Stratford PW, Lott SA, et al. The lower extremity functional scale (LEFS): scale development, measurement properties, and clinical application. North American orthopaedic rehabilitation research network. Phys Ther 1999;79:371-83.

25 Hoogeboom TJ, de Bie RA, den Broeder AA, et al. The Dutch lower extremity functional scale was highly reliable, valid and responsive in individuals with hip/knee osteoarthritis: a validation study. BMC Musculoskelet Disord 2012;13:117.

26 EQ-5D. Available: https://euroqol.org/eq-5d-instruments/eq-5d-5Iabout/ [Accessed 16 Sep 2019].

27 Janssen MF, Pickard AS, Golicki D, et al. Measurement properties of the EQ-5D-5L compared to the EQ-5D-3L across eight patient groups: a multi-country study. Qual Life Res 2013;22:1717-27.

28 Pickwell K, Siersma V, Kars M, et al. Minor amputation does not negatively affect health-related quality of life as compared with conservative treatment in patients with a diabetic foot ulcer: an observational study. Diabetes Metab Res Rev 2017;33. doi:10.1002/ dmrr.2867. [Epub ahead of print: 30 Nov 2016].

29 Van der Heyden J, Charafeddine R. Enquête de santé 2013. Rapport 1: Santé et Bien-être. WIV-ISP, Bruxelles, 2014

30 Schaper NC. Diabetic foot ulcer classification system for research purposes: a progress report on criteria for including patients in research studies. Diabetes Metab Res Rev 2004;20 Suppl 1:S90-5.

31 Mokkink LB, Terwee CB, Knol DL, et al. The COSMIN checklist for evaluating the methodological quality of studies on measurement properties: a clarification of its content. BMC Med Res Methodol 2010;10:22.

32 Heinl D, Prinsen CAC, Drucker AM, et al. Measurement properties of quality of life measurement instruments for infants, children and adolescents with eczema: protocol for a systematic review. Syst Rev 2016;5:25.

33 Checklists for assessing study qualities. Available: https://www. cosmin.nl/tools/checklists-assessing-methodological-study-qualities/

34 Altman DG. Practical statistics for medical research. London: Chapman and Hall, 1991.

35 Kline P. The handbook of psychological testing. London: Routledge, 1993.

36 Siersma V, Thorsen $\mathrm{H}$, Holstein PE, et al. Health-related quality of life predicts major amputation and death, but not healing, in people with diabetes presenting with foot ulcers: the Eurodiale study. Diabetes Care 2014;37:694-700. 
37 Wukich DK, Raspovic KM. Assessing health-related quality of life in patients with diabetic foot disease: why is it important and how can we improve? the 2017 Roger E. Pecoraro Award Lecture. Diabetes Care 2018;41:391-7.

38 Kontodimopoulos N, Veniou A, Tentolouris N, et al. Validity and reliability of the Greek version of the Diabetic Foot Ulcer Scale-Short Form (DFS-SF). Hormones 2016;15:394-403.

39 Spanos K, Saleptsis V, Athanasoulas A, et al. Factors associated with ulcer healing and quality of life in patients with diabetic foot ulcer. Angiology 2017;68:242-50.

40 Holman N, Young B, Stephens H, et al. Pilot study to assess measures to be used in the prospective audit of the management of foot ulcers in people with diabetes. Diabet Med 2015;32:78-84.
41 Li G, Hopkins RB, Levine MAH, et al. Relationship between hyperbaric oxygen therapy and quality of life in participants with chronic diabetic foot ulcers: data from a randomized controlled trial. Acta Diabetol 2017;54:823-31.

42 Siersma V, Thorsen H, Holstein PE, et al. Diabetic complications do not hamper improvement of health-related quality of life over the course of treatment of diabetic foot ulcers - the Eurodiale study. $J$ Diabetes Complications 2017;31:1145-51.

43 Patient-Centered Outcomes Research Institute. Guidance on reporting research on patient-reported outcome measures (PROMs). Available: https://www.pcori.org/research-results/2016/ guidance-reporting-research-patient-reported-outcome-measuresproms 